\title{
Study of in-plane magnetic anisotropy of ultrathin epitaxial Fe films grown on vicinal Mo(110) surface
}

\author{
V. Usov, ${ }^{\text {a) }}$ S. Murphy, and I. V. Shvets \\ SFI Nanoscience Laboratory, Department of Physics, Trinity College, Dublin 2, Ireland
}

(Presented on 8 January 2004)

\begin{abstract}
In situ surface magneto-optical Kerr effect was used in conjunction with scanning tunneling microscopy and low-energy electron diffraction to study the in-plane magnetic anisotropy of ultrathin Fe films on a vicinal Mo(110) substrate. A uniaxial anisotropy with the easy axis of magnetization along the [001] direction was found for this system down to nearly 2 monolayers coverage, contradicting the results of recent theoretical calculations. The easy axis of a step-induced anisotropy coincides with the surface step edges. (C) 2004 American Institute of Physics.
\end{abstract}

[DOI: $10.1063 / 1.1652411]$

\section{INTRODUCTION}

Ultrathin heteroepitaxial films of magnetic $3 d$ transition metals have stimulated much interest as the reduced film thickness and the presence of an heterogeneous interface can substantially modify magnetic properties. As substrates for Fe epitaxial films, Mo(110) surface is very suitable because of its higher surface free energy $\left(\gamma_{\mathrm{Mo}}=2.95 \mathrm{~J} \mathrm{~m}^{-2}\right)^{1}$ and the absence of surface alloying or interdiffusion at temperatures up to $900 \mathrm{~K}^{2}{ }^{2}$ The room-temperature growth of $\mathrm{Fe}$ on Mo(110) has been well studied, ${ }^{2-4}$ but very few data are available on the magnetism of this system. It is known, that for $\mathrm{Mo} / \mathrm{Fe} / \mathrm{Mo}(110)$ multilayers, the in-plane easy axis lies along [001] direction for the Fe layer thickness $7.5 \leqslant d$ $\leqslant 100 \AA$. For the thickness $7.5 \leqslant d \leqslant 10 \AA$, a large in-plane anisotropy of about $500 \mathrm{mT}$ between the [1-10] and [001] directions is present in the system. ${ }^{5}$ In this study, we present details of the anisotropic behavior of uncapped Fe films grown on a vicinal Mo(110) substrate measured under ultrahigh vacuum (UHV) conditions. The choice of a vicinal surface with the step edges between the [001] and [1-10] axes presents the opportunity to determine the preferred direction of magnetization for the step-induced anisotropy from the shift of the anisotropy axes away from the main crystallographic directions. The aim of this study is to measure the in-plane anisotropy and extract the contribution from the atomic terrace steps. To our knowledge, no other in situ measurements of the magnetic properties of the Fe/Mo(110) system have been done to date. Previous experimental data have been obtained ex situ with a metal overlayer on top of the $\mathrm{Fe}$ film.

\section{EXPERIMENT}

The substrate was a $4 \mathrm{~N}$ purity Mo single crystal, with a miscut of $4.6^{\circ}$ from the (110) plane along the [1-1-1] direction. This produced a vicinal $\operatorname{Mo}(110)$ surface with $\sim 25 \AA$ wide terraces separated by single atomic steps [Fig. 1(a)]. The substrate was cleaned in UHV by alternate cycles of

\footnotetext{
${ }^{a)}$ Electronic mail: oussovv@tcd.ie
}

oxidation at $1300 \leqslant T \leqslant 1400 \mathrm{~K}$ in $5 \times 10^{-7}$ Torr of oxygen and subsequent flash annealing to $2300 \mathrm{~K}$ in UHV. This procedure was repeated until the surface impurity levels were below 1 at. $\%$ as determined by Auger electron spectroscopy and a sharp $1 \times 1$ low-energy electron diffraction (LEED) pattern, consistent with the bulk termination of the Mo(110) surface [Fig. 1(b)] was obtained. Fe films were deposited on the substrate at room temperature by electron-beam evaporation of a $4 \mathrm{~N}$ purity $\mathrm{Fe}$ rod. The deposition rates were monitored using a quartz crystal balance and were typically in the $0.005 \leqslant D \leqslant 0.01 \AA / s$ range. The scanning tunneling microscopy (STM) and surface magneto-optical Kerr effect (SMOKE) measurements were performed using home-built instruments.

\section{RESULTS AND DISCUSSION}

STM images of Fe films grown on the Mo(110) substrate at room temperature with a nominal thickness of $2 \AA$ and $5 \AA$ are shown in Fig. 1. At room temperature, the film growth proceeds in the pseudo layer-by-layer mode by the formation of two-dimensional Fe islands. However, the film roughness increases in the thicker films. The corresponding LEED results (not shown here) indicate that the film grows in one-toone registry with the substrate. There is no evidence of dislocation formation in either the LEED or STM data up to a deposited nominal thickness of $8 \AA$.

Longitudinal SMOKE was used to measure the hysteresis loops at $300 \mathrm{~K}$ for different azimuthal orientations of the crystal with respect to the direction of the applied magnetic field. The in-plane sample rotation angle $\varphi$ was measured from the [00-1] crystallographic axis in a counterclockwise direction [Fig. 2(h)]. The normalized angle dependent hysteresis loops for a $10 \AA$ film are displayed in Fig. 2. Qualitatively, films of other thicknesses behaved similarly down to a $5 \AA$ thickness, aside from a Kerr intensity change that generally scaled with the film thickness in the range studied.

Rectangular loops with nearly unitary $M_{r} / M_{s}$ (where $M_{r}$ and $M_{s}$ are the remanent and saturation magnetizations, respectively) like that displayed in Fig. 2(a) were obtained when the magnetic field was applied close to [001] direction, 

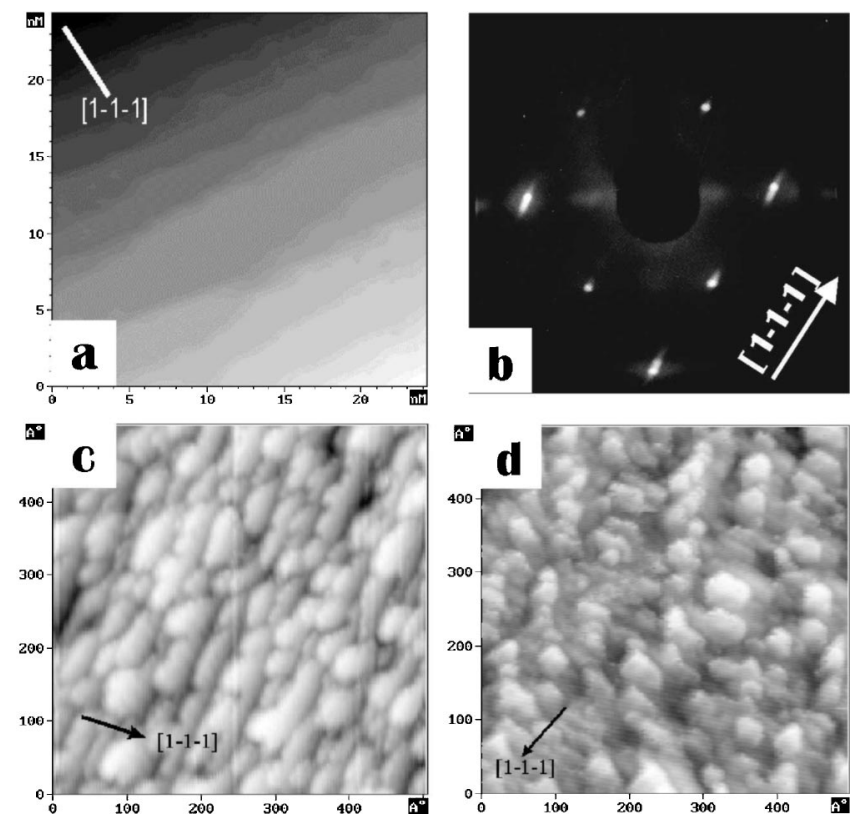

FIG. 1. (a) STM image showing the stepped surface of the clean vicinal Mo(110) substrate; (b) LEED pattern of the clean vicinal Mo(110) surface; (c) $500 \AA \times 500 \AA$ STM image of a $2 \AA$ nominal thickness Fe film grown on the vicinal $\mathrm{Mo}(110)$ surface at room temperature; (d) $500 \AA \times 500 \AA \mathrm{STM}$ image of a $5 \AA$ nominal thickness Fe film grown on Mo(110) at room temperature.

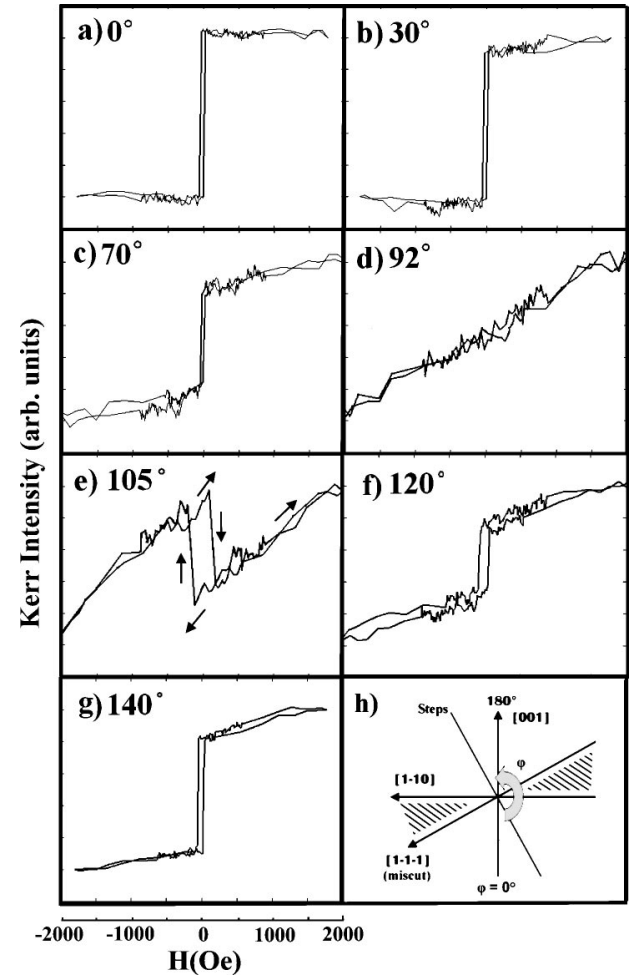

FIG. 2. [(a)-(g)] Normalized in-plane hysteresis loops of a $10 \AA$ Fe film on stepped $\mathrm{Mo}(100)$, measured at $300 \mathrm{~K}$ for different angles $\varphi$; (e) the arrows mark the direction of the Kerr intensity change with increasing $H$; (h) Orientation of $\varphi$ with respect to the $\mathrm{Mo}(110)$ axes. The crosshatch indicates the region of NDM.

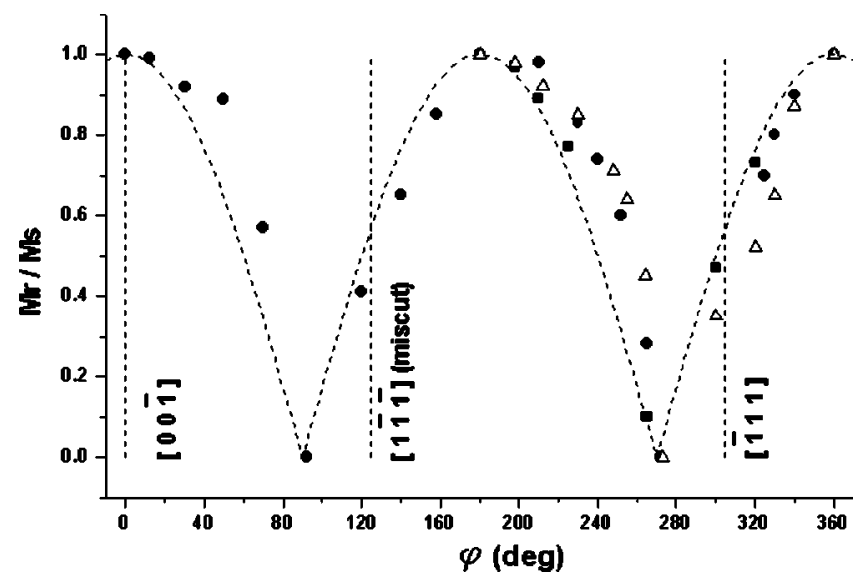

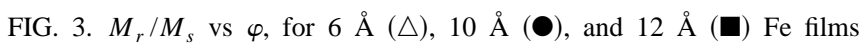
deposited on the $\operatorname{Mo}(110)$ stepped surface. The dashed line corresponds to the $|\cos (\varphi)|$ function.

signifying that the in-plane [001] direction was the easy axis of magnetization of the film. This is exactly opposite to the seemingly similar $\mathrm{Fe} / \mathrm{W}(110)$ system. $^{6}$ Applying the magnetic field nearly along the [1-10] direction [Fig. 2(d)] results in a typical hard axis loops, indicating that a two-fold inplane anisotropy is present in the films (the magnetic field of 2200 Oe is maximum, attainable in our experiment). The dependency of $M_{r} / M_{s}$ on $\varphi$ followed a $\cos (\varphi)$ law. There were deviations from this, which were more pronounced for thinner films (Fig. 3). These resulted from the uniaxial stepinduced anisotropy, caused by the surface miscut, that had both hard and easy axes noncollinear to those of the magnetocrystalline anisotropy. The anisotropy energy density for two noncollinear uniaxial anisotropies with the easy axes at some angle $\alpha$ to each other can be expressed (neglecting second-order uniaxial terms) by

$$
E=K_{1} \sin ^{2}(\theta)+K_{2} \sin ^{2}(\alpha-\theta) .
$$

In our case, $K_{1}$ is the uniaxial anisotropy constant (in $\mathrm{erg} / \mathrm{cm}^{3}$ ) for Fe film on a flat Mo(110) surface with an easy axis of magnetization along the [001] direction, while $K_{2}$ corresponds to the step-induced anisotropy, $\theta$ is an angle between the magnetization vector and the easy axis of the first anisotropy term in Eq. (1). If $\alpha$ is not equal to $90^{\circ}$, then the preferred direction of magnetization of the resulting uniaxial anisotropy lies between the easy axis directions of the first and the second anisotropies. ${ }^{7}$ The shift of the easy axis from the [001] direction toward the steps is obvious from Fig. 3, indicating that the easy axis of the step-induced anisotropy lies along the atomic step edges. No influence of the miscut on the magnetic behavior of the Fe films was observed for the thicknesses above $12 \AA$ due to the loss in the step straightness.

As $M_{r} / M_{s}$ follows the $\cos (\varphi)$ law, the coercive field $H_{c}$ is small compared to the anisotropy field and the magnetization rapidly flips to the opposite direction, we suggest that the magnetization reversal along the easy axis is achieved through the domain-wall movement, while it mainly occurs through the homogeneous rotation along the hard axis. When the magnetic field is applied in any other direction, the re- 
versal process is a combination of these two mechanisms. However, this is not valid if the external field is applied between the [1-10] and [1-1-1] (miscut) directions. In this case, the hysteresis loops [Fig. 2(e)] reveal a region of negative differential magnetization (NDM) [shaded in Fig. 2(h)]. When the applied field changes from negative to positive, the magnetization reaches a local maximum and spontaneously flips back to the negative direction. A further magnetization reversal process continues by means of the coherent rotation. Hence, we observed NDM in a single-material ultrathin film on a (110) stepped surface. More detailed discussion on this phenomenon is a subject of a following publication.

In contrast to the $\mathrm{Mo} / \mathrm{Fe}(7.5 \AA) / \mathrm{Mo}(110)$ sample that was found to be superparamagnetic at $300 \mathrm{~K},{ }^{5} \mathrm{Fe} / \mathrm{Mo}(110)$ remained ferromagnetic down to $5 \AA$. A $3.7 \AA$ Fe film did not show any sign of ferromagnetic behavior at room temperature, but produced an easy axis loop along the [001] axis below $250 \mathrm{~K}$. This disagrees with the results of recent theoretical calculations, ${ }^{8}$ predicting an easy axis of magnetization along the [1-10] direction. The dramatic rise of $H_{c}$ from 100 to about 3000 Oe reported for the $\mathrm{Mo} / \mathrm{Fe}(7.5 \AA) / \mathrm{Mo}(110)$ system $^{5}$ has never been observed in our experiments and is rather the result of the presence of the additional $\mathrm{Fe} / \mathrm{Mo}(110)$ interface than an intrinsic property of the $\mathrm{Fe} / \mathrm{Mo}(110)$ system.
In conclusion, Fe films have been epitaxially grown on a vicinal Mo(110) single crystal. The Fe/Mo(110) system has an uniaxial anisotropy with the easy axis of magnetization along the [001] direction. The system also reveals a two-fold step-induced anisotropy with the easy axis of magnetization along the atomic steps. The hysteresis loops with NDM have been produced when the external field is applied between the [1-1-1] axis and the direction perpendicular to the step edges.

\section{ACKNOWLEDGMENT}

This work was supported by Science Foundation of Ireland (SFI) under Contract No. 00/PI.1/C042.

${ }^{1}$ L. Mezey and J. Giber, Surf. Sci. 117, 220 (1982).

${ }^{2}$ M. Tikhov and E. Bauer, Surf. Sci. 232, 73 (1990).

${ }^{3}$ J. Malzbender, M. Przybylski, J. Giergiel, and J. Kirschner, Surf. Sci. 414, 187 (1998).

${ }^{4}$ S. Murphy, D. Mac Mathuna, G. Mariotto, and I. V. Shvets, Phys. Rev. B 66, 195417 (2002).

${ }^{5}$ O. Fruchart, J. P. Nozières, and D. Givord, J. Magn. Magn. Mater. 207, 158 (1999).

${ }^{6}$ U. Gradmann, J. Korecki, and G. Waller, Appl. Phys. A: Solids Surf. 39, 101 (1986).

${ }^{7}$ B. D. Cullity, in Introduction to Magnetic Materials, edited by M. Cohen (Addison-Wesley, Reading, MA, 1972), p. 246.

${ }^{8}$ X. Qian and W. Hubner, Phys. Rev. B 64, 092402 (2001). 